\title{
THE EFFECTS OF THE CROSS-SECTIONAL AREA AND ASPECT RATIO OF BEAMS ON A LAMINAR CHANNEL FLOW
}

\author{
Hai-Ping Hu \\ Department of Marine Engineering, National Taiwan Ocean University, Keelung, Taiwan. \\ Rong-Hua Yeh \\ Department of Marine Engineering, National Kaohsiung Marine University, Kaohsiung, 81143, Taiwan.
}

Follow this and additional works at: https://jmstt.ntou.edu.tw/journal

Part of the Engineering Commons

\section{Recommended Citation}

Hu, Hai-Ping and Yeh, Rong-Hua (2010) "THE EFFECTS OF THE CROSS-SECTIONAL AREA AND ASPECT RATIO OF BEAMS ON A LAMINAR CHANNEL FLOW," Journal of Marine Science and Technology. Vol. 18: Iss. 2, Article 4. DOI: $10.51400 / 2709-6998.2317$

Available at: https://jmstt.ntou.edu.tw/journal/vol18/iss2/4

This Research Article is brought to you for free and open access by Journal of Marine Science and Technology. It has been accepted for inclusion in Journal of Marine Science and Technology by an authorized editor of Journal of Marine Science and Technology. 


\title{
THE EFFECTS OF THE CROSS-SECTIONAL AREA AND ASPECT RATIO OF BEAMS ON A LAMINAR CHANNEL FLOW
}

\author{
Hai-Ping $\mathrm{Hu}^{*}$ and Rong-Hua Yeh**
}

Key words: viscous flow, eigenfunction, point-matched, beam.

\begin{abstract}
The study analyzed the fully developed laminar flow in a channel with longitudinal rectangular beams arrayed along the channel width. The governing equations describing the fluid which flows along the direction of the beam length are expressed with double Poisson equations and are solved by eigenfunction-expansion and point-match method. The effects of cross-sectional area and aspect ratio of the beams are investigated. Dimensionless velocity profile, mean velocity, and friction factor multiplied by Reynolds number in the channel are presented for analysis. Initially, a comparison of $f R e$ for a special case is made. It is found that the predicted $f R e$ of this work tends to approach that of a parallel-plate channel case for smaller $A$ and $a_{\mathrm{r}}$. A further study shows that increasing the cross-sectional area of the beams will lead to a decrease both in the mean velocity and $f R e$ values. For a fixed cross-section area of the beam, as the beam height increases, an increase in the mean velocity and volumetric flow rate of the fluid but a decrease in the $f R e$ is obtained.
\end{abstract}

\section{INTRODUCTION}

The channel flow plays an important role in the research and analysis of fluid mechanics. Nowadays, more and more attention has been engrossed on flow in a channel with rectangular beams. Practical engineering applications can be found in galvanizing and coating process on the bars, designing a lubrication system, and cooling elements in a mechanical device. The pressure drop in this process can be obtained from the calculation of flow velocity and friction factor in the channel. Consequently, many significant efforts directed towards researches with related fields. Sparrow and

Paper submitted 11/14/08; revised 04/08/09; accepted 04/19/09. Author for correspondence: Rong-Hua Yeh (e-mail: rhyeh@mail.nkmu.edu.tw).

*Department of Marine Engineering, National Taiwan Ocean University, Keelung, Taiwan.

**Department of Marine Engineering, National Kaohsiung Marine University, Kaohsiung, 81143, Taiwan.
Loeffler [8] investigated the laminar flow between cylinders arranged in regular array by using the eigenfunction-expansion and point-matched method. Their analytical solution was obtained for the longitudinal fully developed laminar flow between cylinders arranged in triangular of square array. Cheng [3] also presented an analog solution of laminar heat transfer in noncircular ducts by point-matching. The fully developed laminar flow characteristics in noncircular ducts and the steady temperature distribution in infinitely long prismatic bars can be obtained. Later, neglecting inertia effect, Trogdon and Joseph [9] solved the problem of plane flow of a second-order fluid over a rectangular slot by matching biorthogonal eigenfucntion expansions in different regions of flow. Besides, some other efforts directed studies towards a channel with various cross section such as a complex shaped duct or channel, in which the complicated regions might be decomposed into contiguous simpler sub-regions. In 1976, Zarling [12] proposed the semi-analytical method to solve the governing equation for fully developed laminar flow through bars of complex geometrical pattern. Employing the SchwarzNeumann alternating method along with least squares point matching, the flow velocity in a duct with one pair of opposing walls being parallel plates and the adjacent pair of walls semicircular arcs was obtained. In addition, the method was applied to a complex shaped duct and the resulting velocity was used to calculate the flow rate and pressure drop. Meanwhile, Wang [10] analyzed the parallel flow between two fixed corrugated plates, and discussed the dependence of flow rate on effect of wavy surface. In 1994, Wang [11] further studied the laminar, viscous flow between parallel plates with evenly spaced longitudinal ribs. The flow in a channel with longitudinal ribs was solved by an eigenfunction-expansion and point-match method. It was found that both the wetted perimeter and the friction factor-Reynolds number product are unsuitable parameters for the flow through ducts of complex geometry. For a circular cylinder subject to a parallel-plate channel, Chen et al. [2] investigated the steady flow past this cylinder lost stability as the Reynolds number was increased. Furthermore, Bahrami et al. [1] investigated the fully-developed laminar flow in smooth channels of arbitrary cross-sections. For some selected channels, the $f R e$ values were computed using existing analytical solutions for fluid flow. Recently, 


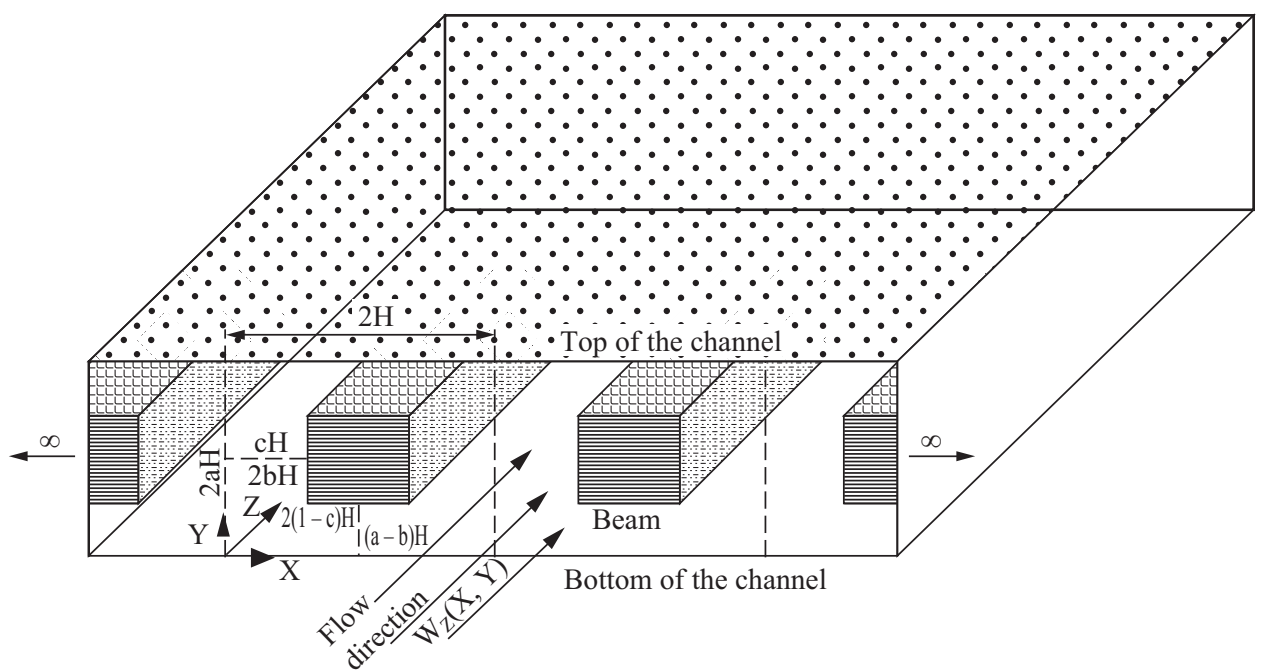

(a)

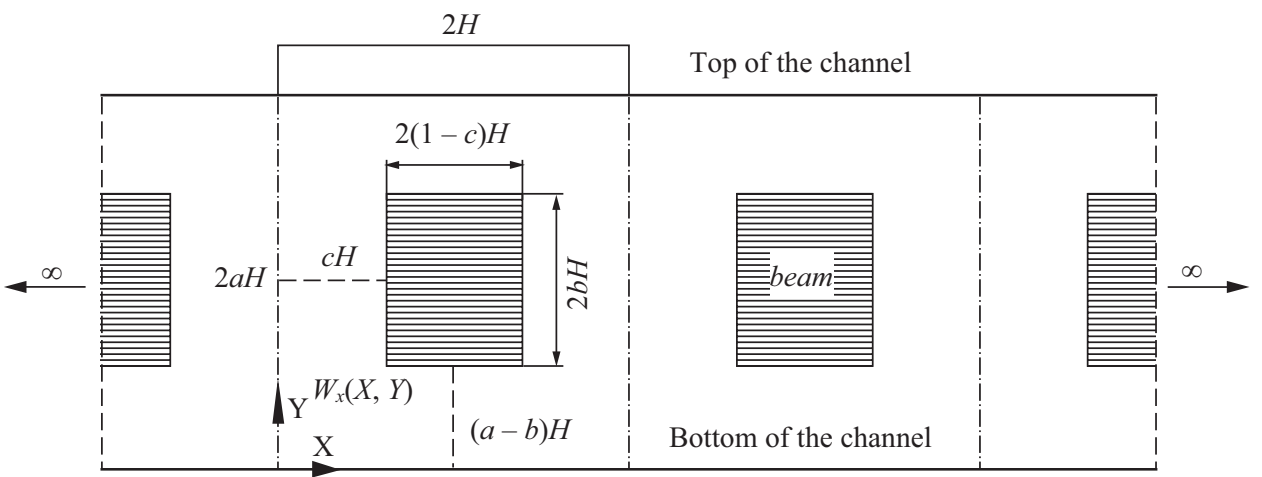

(b)

Fig. 1. (a) The overall view of the proposed model, (b) The X-Y cross-section of the channel with rectangular beams.

Hsieh and Chen [6] investigated the emergence and development of cylinder-attached recirculation regions in a fully developed flow past a circular cylinder which was symmetrically confined between two flat plates.

Although many researches about channel flow under different conditions were discussed, the channel flow with arrayed beams of different aspect ratios and cross-sectional areas is also worth discussing particularly in the thermal and flow analysis of metallic bars under coating and galvanizing process. The present paper, thus, investigates the laminar channel flow passing a complex geometric configuration, i. e. a channel including rectangular beams positioned along the $\mathrm{X}$-direction with flow directing in Z-direction. In addition, local dimensionless velocity, mean velocity and friction factor-Reynolds number product are obtained for various sizes of beams in a channel.

\section{ANALYSIS}

Consider a steady state, fully developed laminar channel flow between the upper and the lower plate. The height of the channel is $2 a H$. Assume both the left and the right sides of the channel extend to infinity. Inside the channel, a series of beams were arrayed along the $X$-direction. The height of beam is $2 b H$ and the width of the beam is equal to $2(1-c) H$. The overall view of the physical model and cross-section of the channel are shown in the Figs. 1(a) and 1(b) respectively. Note that flow direction is normal to the paper and is in the positive Z-direction. Since the flow velocity is very slow, negligible inertia force is assumed. For a steady and fully developed laminar flow, Navier-Stoke's equation describing a three-dimensional i.e. $\frac{\partial W}{\partial t}=0, U=V=0, \frac{\partial W}{\partial Z}=0$, amd $\frac{\partial^{2} W}{\partial Z^{2}}=0$, the flow can then be simplified as

$$
0=\rho g_{z}-\frac{d p}{d Z}+\mu\left(\frac{\partial^{2} W}{\partial X^{2}}+\frac{\partial^{2} W}{\partial Y^{2}}\right)
$$

Viewing Fig. 1(a), $\rho g_{z}$ vanishes because the gravity acceleration is in the negative y direction and the governing equation can be expressed as: 


$$
\nabla^{2} W(X, Y)=\frac{1}{\mu} \frac{d p}{d Z}=\text { const }
$$

Further introduce the dimensionless parameter $w=$ $-W /\left(\frac{H^{2} d p}{\mu d Z}\right)$ into (2), and the governing equation can be rewritten in the dimensionless form of Poisson equation:

$$
\nabla^{2} w(x, y)=-1
$$

Consider the domain with a period, $2 H$, and focused on the range from $x=0$ to $x=H$, and $y=0$ to $y=a H$, as shown in Fig. 1(b). The range is symmetric to y-axis in $x=0$ and symmetric to $\mathrm{x}$-axis in $y=a H$. Due to symmetry, only one part of the channel, an L-Shaped region with two combined rectangles, as displayed in Fig. 2, is taken into account. For convenience of reading and analysis, the L-shaped region in Fig. 1(b) is magnified into Fig. 2 with the height and width given in a dimensionless form. The dimensionless governing equation for the left rectangle of the L-shaped domain is:

$$
\nabla^{2} w_{1}(x, y)=-1
$$

and the corresponding boundary conditions are:

$$
w_{1}(x, 0)=0, \frac{\partial w_{1}}{\partial y}(x, a)=0, \frac{\partial w_{1}}{\partial x}(0, y)=0
$$

Next, the flow equation of the right rectangle in the L-shaped domain can be expressed as:

$$
\nabla^{2} w_{2}(x, y)=-1
$$

subject to the boundary conditions below:

$$
w_{2}(x, 0)=0, w_{2}(x, a-b)=0, \frac{\partial w_{2}}{\partial x}(1, y)=0
$$

With the boundary conditions of (5) and (7), Eqs. (4) and (6) can be solved and obtained as follows:

$$
\begin{aligned}
& w_{1}(x, y)=y\left(a-\frac{y}{2}\right)+\sum_{n} A_{n} \sin \left(\alpha_{n} y\right)\left(e^{\alpha_{n}(x-c)}+e^{-\alpha_{n}(x+c)}\right) \\
& w_{2}(x, y)=\frac{y}{2}(a-b-y)+\sum_{m} B_{m} \sin \left(\beta_{m} y\right)\left(e^{\beta_{m}(x-2+c)}+e^{-\beta_{m}(x-c)}\right)
\end{aligned}
$$

where the eigenvalues respectively are:

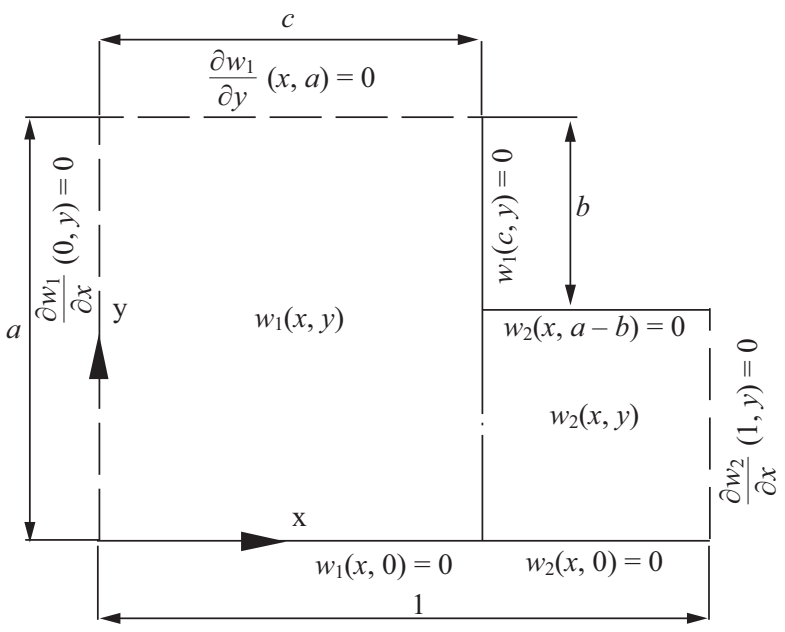

Fig. 2. Enlarged view of the L-shape regions with dimensionless parameters.

$$
\alpha_{n}=\frac{(2 n-1) \pi}{2 a} \text { and } \beta_{m}=\frac{m \pi}{a-b}
$$

One more boundary condition for (4) is:

$$
w_{1}(c, y)=0 ; a-b \leq y \leq a
$$

Substitute (11) into (8), and the following equation can be obtained:

$$
\begin{array}{r}
\sum_{n} A_{n} \sin \left(\alpha_{n} y_{i}\right)\left(1+e^{-2 \alpha_{n} c}\right)=y_{i}\left(\frac{y_{i}}{2}-a\right) \\
\mathrm{i}=\mathrm{M}+1 \text { to } \mathrm{N}
\end{array}
$$

It should be pointed out that the traditional direct integral methods fail to solve the proposed problem on account of being short of the boundary condition in the range of $0 \leq y<$ $a-b$. However, the point-matched method can tackle this problem without much difficulty. Subsequently, the velocity and the shear stress of the two rectangle regions of the Lshaped domain can be matched along the common boundary by using the point-matched method [4, 7]. The common boundary conditions are given as:

$$
\begin{gathered}
w_{1}(c, y)=w_{2}(c, y), 0 \leq y<a-b \\
\frac{\partial w_{1}(c, y)}{\partial x}=\frac{\partial w_{2}(c, y)}{\partial x}, 0 \leq y<a-b
\end{gathered}
$$

Substituting the boundary conditions (13) and (14) into (8) and (9), it yields: 
Table 1. The effects of $\Delta x$ on $w(0,1)$.

\begin{tabular}{cccccc}
\hline & \multicolumn{5}{c}{$\mathrm{A}=0.15, \mathrm{~b}=0.25, \mathrm{c}=0.4$} \\
\cline { 2 - 6 } $\begin{array}{c}\Delta x \\
w(0,1)\end{array}$ & 0.03 & 0.02 & 0.01 & 0.005 & 0.001 \\
\cline { 2 - 6 } & 0.3997 & 0.4146 & 0.4195 & 0.4195 & 0.4196 \\
\cline { 2 - 6 } & & & $\mathrm{A}=0.2, \mathrm{~b}=0.4, \mathrm{c}=0.5$ & 0.005 & 0.001 \\
\cline { 2 - 6 } & 0.03 & 0.02 & 0.01 & 0.1179 & 0.1180 \\
\hline
\end{tabular}

$$
\begin{aligned}
\sum_{n} A_{n} \sin \left(\alpha_{n} y_{i}\right)\left(1+e^{-2 c \alpha_{n}}\right)-\sum_{m} B_{m} \sin \left(\beta_{m} y_{i}\right)\left(1+e^{-2(1-c) \beta_{m}}\right) \\
=-\frac{(a+b)}{2} y_{i}
\end{aligned}
$$$$
\mathrm{i}=1 \text { to } \mathrm{M}(15)
$$

$$
\begin{aligned}
\sum_{n} A_{n} \alpha_{n} \sin \left(\alpha_{n} y_{i}\right)\left(1-e^{\left.-2 c \alpha_{n}\right)}\right) & \\
+\sum_{m} B_{m} \beta_{m} \sin \left(\beta_{m} y_{i}\right)\left(1-e^{-2(1-c) \beta_{m}}\right) & =0 \\
\mathrm{i} & =1 \text { to } \mathrm{M}(16)
\end{aligned}
$$

The mean value for $w(x, y)$ is derived as:

$$
w_{\text {mean }}=\frac{1}{a-b(1-c)}\left[\int_{0}^{c} \int_{0}^{a} w_{1} d y d x+\int_{c}^{1} \int_{0}^{a-b} w_{2} d y d x\right]
$$

Integrating (17), it gives:

$$
\begin{aligned}
w_{\text {mean }}= & \frac{1}{a-b(1-c)} \\
& \left\{\begin{array}{l}
\sum_{n} A_{n} \frac{1}{\alpha^{2}}(1-\cos n \pi)\left(1-e^{-2 c \alpha}\right)+\frac{(1-c)(a-b)^{3}}{12} \\
+\sum_{m} B_{m} \frac{1}{\beta^{2}}[\cos (a-b) \beta-1]\left[e^{-2 \beta(1-c)}-1\right]+\frac{c a^{3}}{3}
\end{array}\right\}
\end{aligned}
$$

The volumetric flow rate, $q$, of the fluid in the channel is defined as the mean value of $w(x, y), w_{\text {mean }}$, multiplied the cross-sectional area of the flow.

Furthermore, the $f \cdot \operatorname{Re}$ can be expressed as the function of $w_{\text {mean }}$ as described in Wang [11] and is written below:

$$
f \cdot \operatorname{Re}=\frac{D_{h}^{2}}{2 w_{\text {mean }} H^{2}}
$$

or in a more detailed form,

$$
f \cdot \operatorname{Re}=\frac{8[a-b(1-c)]^{2}}{(2+b-c)^{2} w_{\text {mean }}}
$$

where $D_{h}$ is the hydraulic diameter.

\section{NUMERICAL METHOD}

Equations (8) and (9) can be used to estimate $w_{1}(x, y)$ and $w_{2}(x, y)$ by using Visual $\mathrm{C}^{++}$. The detailed steps of numerical methods are given as follows:

(1) Specify the constant $c, A$, and $N$.

(2) Set $\mathrm{M}=$ floor $[(1-\mathrm{b} / \mathrm{a})]+1$ and $y_{i}=i a / N$.

(3) Express (12), (15) and (16) in a linear system of $(N+M)$ equations which can be used to solve coefficients $A_{n}$ and $B_{m}$.

(4) Substitute coefficients $A_{n}$ and $B_{m}$ into (8) and (9).

(5) Set $\Delta y=\frac{a}{N}, y=\Delta y \times i, \Delta x=0.01, x=\Delta x \times j$. Note that for accuracy the effects of $\Delta x$ on the maximum velocity, $w_{1}(x$, $y$ ), are listed in Table 1. This calculating process is repeated at the next node position. The nodes in the range for $w_{1}(x, y)$ are $0 \leq y \leq a$, and $0 \leq x \leq 1-c$, and in the range for $w_{2}(x, y)$ are $0 \leq y \leq a-b$, and $1-c<x \leq 1$.

(6) Map the L-shaped region on the entire channel.

(7) Then, calculate the mean values of the velocity, $q$ and $f \cdot$ Re.

\section{RESULTS AND DISCUSSIONS}

For brevity, a square cross-section area is taken into account, i.e. $a$ is assumed to be unity, throughout this section. Table 2 presents the calculated results of the maximum velocity under different $N$. It shows that convergence is fairly fast for $10 \leq N$ $\leq 40$, and the deviation under the four cases of $N$ is very small. Note that the position of the maximum velocity falls on $(0,1)$ due to the largest space for flow. It is shown the value of $N=$ 30 is accurate enough to be employed in this study. In addition, for convenience of the verification for the prospective work, the coefficients $A_{n}$ and $B_{m}$ of $w_{1}(x, y)$ and $w_{2}(x, y)$ are provided in Table 3. 
Table 2. The influence of $b$ on maximum velocity, $w_{1}(0,1)$, for $a=1$ and $A=0.25$.

\begin{tabular}{ccccccccc}
\hline$b$ & 0.3 & 0.4 & 0.5 & 0.6 & 0.7 & 0.8 & 0.9 & 1.0 \\
\hline$N=10$ & 0.0502 & 0.0702 & 0.1183 & 0.1481 & 0.1709 & 0.1872 & 0.2004 & 0.2108 \\
\hline$N=20$ & 0.0503 & 0.0707 & 0.1175 & 0.1478 & 0.1703 & 0.1872 & 0.2004 & 0.2108 \\
\hline$N=30$ & 0.0503 & 0.0709 & 0.1174 & 0.1477 & 0.1703 & 0.1872 & 0.2004 & 0.2108 \\
\hline$N=40$ & 0.0503 & 0.0709 & 0.1174 & 0.1477 & 0.1703 & 0.1872 & 0.2004 & 0.2108 \\
\hline
\end{tabular}

Table 3. The coefficients of $A_{n}$ and $B_{m}$ for $A=0.1, a=1, b=0.5$.

\begin{tabular}{|c|c|c|c|c|c|c|c|}
\hline \multicolumn{8}{|c|}{$\mathrm{A}_{1} \sim \mathrm{A}_{40}$} \\
\hline $\mathrm{A}_{1}=-0.78941$ & $\mathrm{~A}_{2}=0.31274$ & $\mathrm{~A}_{3}=-0.26570$ & $\mathrm{~A}_{4}=0.21267$ & $\mathrm{~A}_{5}=-0.16644$ & $\mathrm{~A}_{6}=0.11494$ & $\mathrm{~A}_{7}=-0.08280$ & $\mathrm{~A}_{8}=0.07104$ \\
\hline $\mathrm{A}_{9}=-0.05644$ & $\mathrm{~A}_{10}=0.05253$ & $\mathrm{~A}_{11}=-0.04609$ & $\mathrm{~A}_{12}=0.04465$ & $\mathrm{~A}_{13}=-0.03371$ & $\mathrm{~A}_{14}=0.03555$ & $\mathrm{~A}_{15}=-0.00367$ & $\mathrm{~A}_{16}=0.00799$ \\
\hline $\mathrm{A}_{17}=-0.00273$ & $\mathrm{~A}_{18}=0.00466$ & $\mathrm{~A}_{19}=-0.00193$ & $\mathrm{~A}_{20}=0.00371$ & $\mathrm{~A}_{21}=-0.00067$ & $\mathrm{~A}_{22}=0.00259$ & $\mathrm{~A}_{23}=-0.00041$ & $\mathrm{~A}_{24}=0.00182$ \\
\hline $\mathrm{A}_{25}=-0.00018$ & $\mathrm{~A}_{26}=0.00069$ & $\mathrm{~A}_{27}=-0.00000$ & $\mathrm{~A}_{28}=0.00004$ & $\mathrm{~A}_{29}=-0.00000$ & $\mathrm{~A}_{30}=0.00000$ & $\mathrm{~A}_{31}=-0.00000$ & $\mathrm{~A}_{32}=-0.00000$ \\
\hline $\mathrm{A}_{33}=-0.00000$ & $\mathrm{~A}_{34}=0.00000$ & $\mathrm{~A}_{35}=-0.00000$ & $\mathrm{~A}_{36}=0.00000$ & $\mathrm{~A}_{37}=-0.00000$ & $\mathrm{~A}_{38}=0.00000$ & $\mathrm{~A}_{39}=-0.00000$ & $\mathrm{~A}_{40}=0.00000$ \\
\hline \multicolumn{8}{|c|}{$\mathrm{B}_{1} \sim \mathrm{B}_{20}$} \\
\hline $\mathrm{B}_{1}=-0.05870$ & $\mathrm{~B}_{2}=0.00790$ & $\mathrm{~B}_{3}=-0.00146$ & $\mathrm{~B}_{4}=0.00524$ & $\mathrm{~B}_{5}=-0.00112$ & $\mathrm{~B}_{6}=0.00422$ & $\mathrm{~B}_{7}=-0.00092$ & $\mathrm{~B}_{8}=0.00261$ \\
\hline $\mathrm{B}_{9}=-0.00003$ & $\mathrm{~B}_{10}=0.00007$ & $\mathrm{~B}_{11}=-0.00001$ & $\mathrm{~B}_{12}=-0.00003$ & $\mathrm{~B}_{13}=-0.00000$ & $\mathrm{~B}_{14}=0.00000$ & $\mathrm{~B}_{15}=-0.00000$ & $\mathrm{~B}_{16}=0.00000$ \\
\hline $\mathrm{B}_{17}=-0.00000$ & $\mathrm{~B}_{18}=0.00000$ & $\mathrm{~B}_{19}=-0.00000$ & $\mathrm{~B}_{20}=0.00000$ & & & & \\
\hline
\end{tabular}

Figure 3 depicts the local velocity profile in the channel for $a=1.0, b=0.5$ and $A=0.25$. As can be clearly seen in the figures, the local velocities of the bottom and the top of the channel are all zero under the no-slip boundary conditions and the velocity gradually increases away from the fixed boundary. The maximum value of $w$ falls on $w_{1}$ and occurs at $x=0, y=a$. In addition, the maximum $w$ then gradually decreases to zero on the solid boundaries, i.e. the top and the bottom of the channel and the boundary of the beams. At a fixed $A$, the local velocity between the two neighboring beams increases as the beam height increases. This is due to the fact that spacing between beams becomes larger for a higher $b$. Consequently, the boundary layer effect is smaller under this circumstance. To further explain this phenomenon, the local velocity distribution in the channel for $a=1.0, b=0.7$ and $A=0.25$ is given in Fig. 4. Compared with Figs. 3 and 4, it is apparent that the maximum velocity for $b=0.7$ is greater than that for $b=$ 0.5 at $A=0.25$. Also note that the velocity between the beam and the top or bottom plate is larger for a smaller beam height. This can be apprehended that the larger the spacing between beams is, the smaller the spacing between beam and top/ bottom plate of the channel will become at a fixed $A$. For a larger $b$, the clearances between beams and plates are smaller and are almost completely subject to boundary layers which will reduce the flow velocity to a certain extent. However, this phenomenon is alleviated for the beams with smaller $b$. This is because most of the fluid moves through the spacing between beams whereas smaller flow rate of the fluid passes the clearance between beam and top/bottom plate due to the fluid on different beam height and width under the same crosssectional area of the beam.

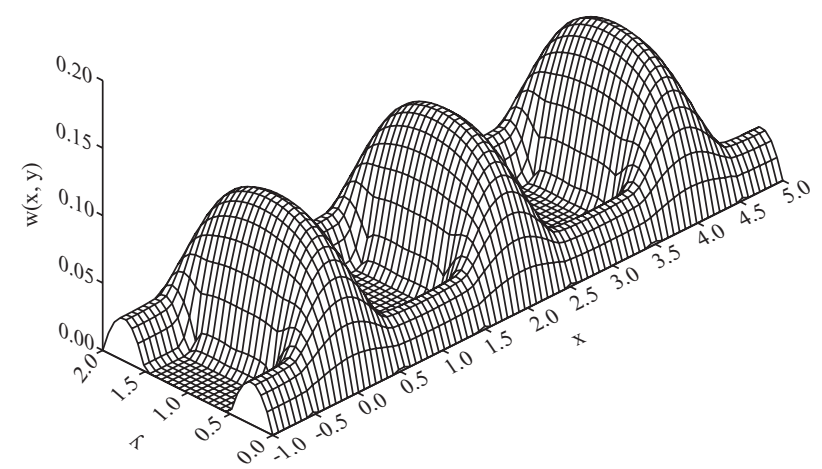

Fig. 3. Local velocity distribution for $a=1.0, b=0.5$ and $A=0.25$.

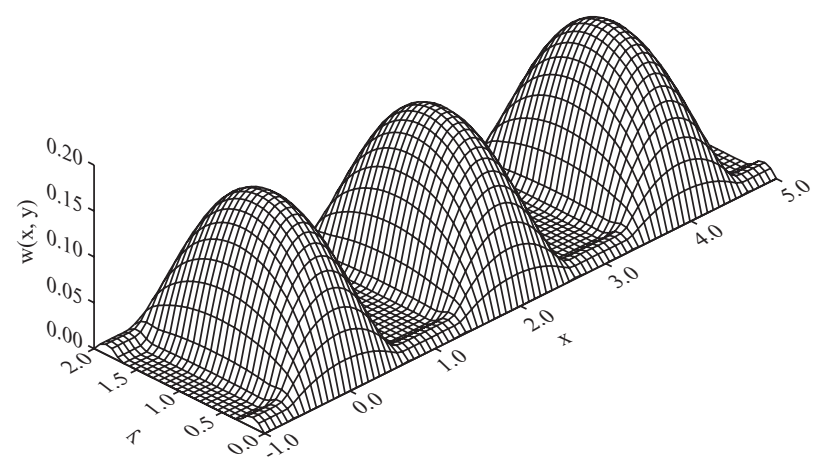

Fig. 4. Local velocity distribution for $a=1.0, b=0.7$ and $A=0.25$.

Figure 5 plots the effect of volumetric flow rate of the aspect ratio of the beam is defined as the ratio of height to width. To normalize the aspect ratio, a parameter of $a_{\mathrm{r}}$ is obtained as: 


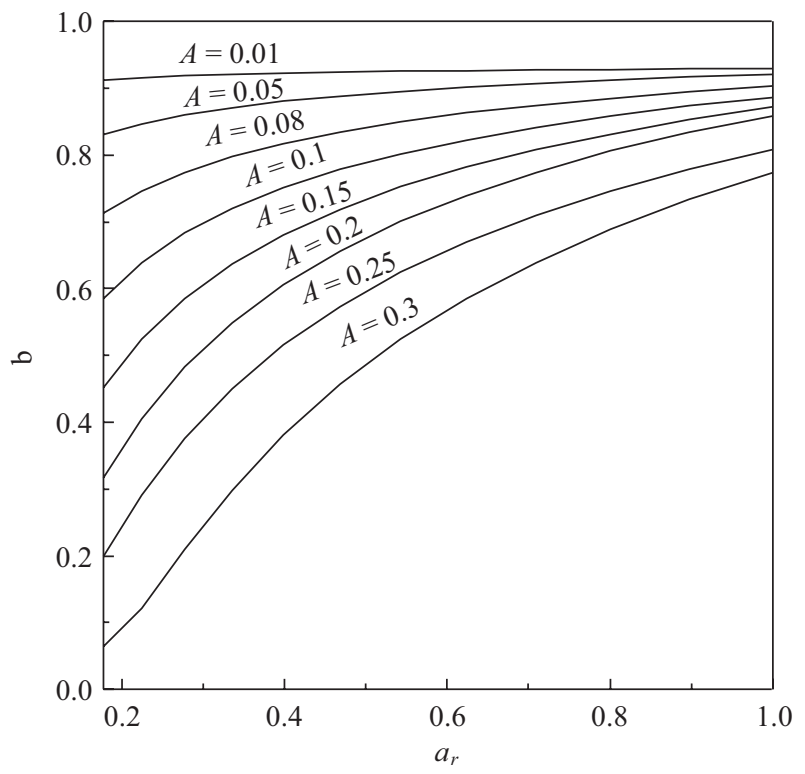

Fig. 5. Volumetric flow rates of the fluid on different aspect ratios.

$a_{r}=\frac{b /(1-c)}{[b /(1-c)]_{\max }}$ or, in a alternative form,

$$
a_{r}=\frac{b /(1-c)}{1 / A}
$$

Note that $[b /(1-c)]_{\max }=1 /(1-c)$ and $b(1-c)=1(1-c)=A$ since $0<b \leq 1$. Moreover, there is a close relationship between $a_{r}$ and $A$. After some mathematical manipulation, it can be derived as:

$$
\sqrt{a_{r}} \geq A>0
$$

Details of the derivation are given in the Appendix of the manuscript. In this case, $A$ must be positive and $A<0.316$ merely exists for $a_{r}=0.1$. This is the reason why $A=0.01$ and 0.3 are selected for demonstration in this figure. It is worthwhile to note that the volumetric flow rate of the flow approaches a constant value as $A$ tends to zero. This can be explained that the situation just like a channel flow without any beams for trivial $A$. Under this circumstance, the volumetric flow rate is invariable despite the variation in $a_{r}$. Furthermore, it is observed that $q$ increases with $a_{r}$ at a fixed $A$. For $a_{r}<0.6$, the increasing rate of the volumetric flow rate of fluid is higher for a larger $A$ whereas a smaller increase in $q$ is obtained for a smaller $A$. As for $a_{r}>0.6$, with an increase in $a_{r}$, the raise in $q$ is insignificant particularly for a smaller $A$. This is because an increase in the cross-sectional area of the beam will lead to a decrease in the flow area, and then consequently bring about a reduction in $q$. In addition, a further explanation of this phenomenon is given in Figs. 6(a) and 6(b). It is

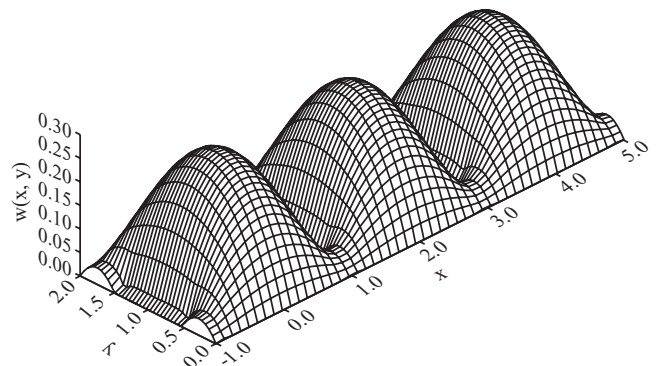

(a)

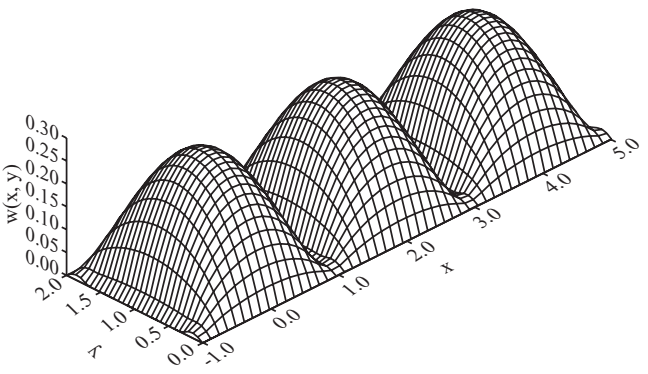

(b)

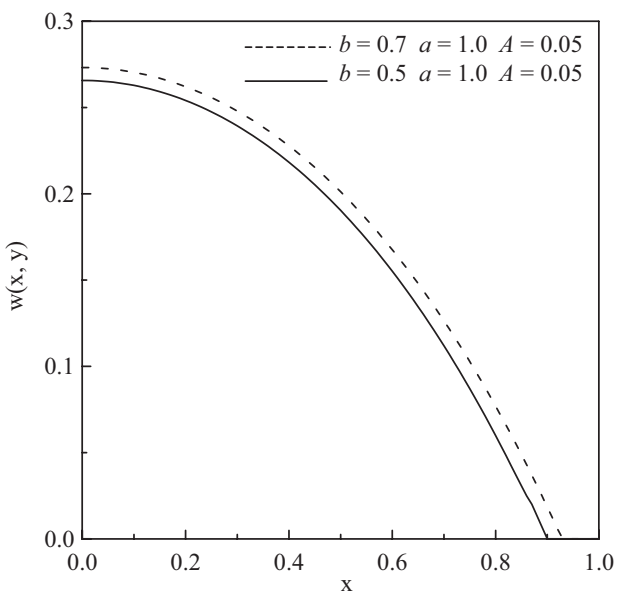

(c)

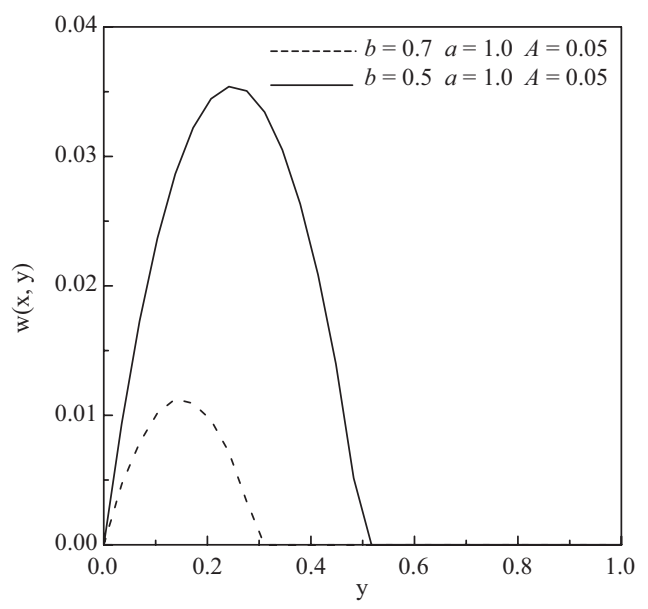

(d)

Fig. 6. (a) Local velocity distribution for $a=1.0, b=0.5$ and $A=0.05$. (b) Local velocity distribution for $a=1.0, b=0.7$ and $A=0.05$. (c) The cross-sectional view of $w_{1}(x, y)$ at $y=1$. (d) The crosssectional view of $w_{2}(x, y)$ at $x=-1$. 


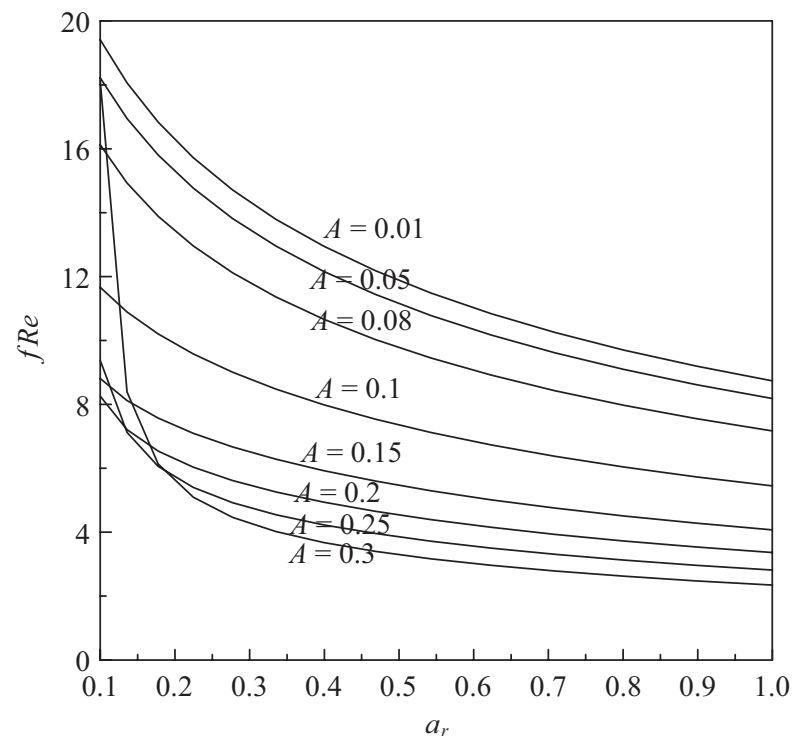

Fig. 7. Effects of $a_{r}$ on $f R e$ for various $A$ 's.

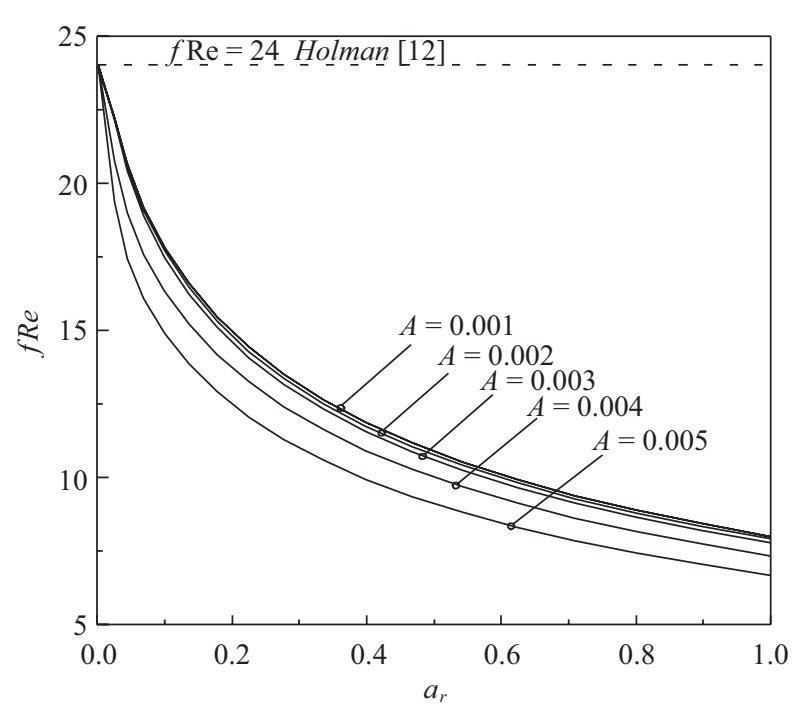

Fig. 8. Dependence of $f R e$ on $a_{\mathrm{r}}$ for smaller $A$.

obtained that $\left(w_{1}\right)_{\max }=0.265$ for $b=0.5$ and $\left(w_{1}\right)_{\max }=0.273$ for $b=0.7$ under the conditions of $A=0.05$ and $a=1$. A closer look of the comparison in $\left(w_{1}\right)_{\max }$ is illustrated in Fig. 6(c). Also, with the observations of Figs. 3 and 4, as $b$ increases from 0.5 to 0.7 , the maximum $w_{1}$ varies from 0.117 to 0.170 for $A=0.25$ and $a=1$. Apparently, a larger cross-sectional area of the beam causes a decrease in $w_{1}$ and then a smaller volumetric flow rate of the fluid is obtained. In addition, an increase in the aspect ratio (i. e. increasing the height of beam, $b)$ will cause an increase in $w_{1}$ and a decrease in $w_{2}$. This is clearly seen in Fig. 6(d) that $\left(w_{2}\right)_{\max }=0.036$ for $b=0.5$ and $\left(w_{2}\right)_{\max }=0.012$ for $b=0.7$ under the conditions of $A=0.05$ and $a=1$. Viewing the increase in $\left(w_{1}\right)_{\max }$ and the decrease in $\left(w_{2}\right)_{\max }$ on increasing $a_{\mathrm{r}}$, it is understood that the influence of $w_{2}$ is quite insignificant. Apparently, $w_{1}$ is decisive in determining $w_{\text {mean }}$ and $q$; and in consequence an increase in $a_{r}$ will bring out an increase in both $w_{\text {mean }}$ and $q$.

Figure 7 depicts the product of the friction factor and Reynolds number for different aspect ratios of beams with the same cross-sectional area. It is shown that $f R e$ decreases as the aspect ratio of the beam increases at a specified $A$. Besides, an increase in the cross-sectional area of the beams will result in a decrease in the $f R e$ at a given $a_{r}$, as expected. Note that there is an abrupt increase in $f R e$ as $a_{r}$ approaches the critical $a_{\mathrm{r}}$ for corresponding $A$, as pointed out in (21). A locally maximum $f R e$ occurs on the critical value $a_{r}$. Apparently, the maximum $f R e$ is dependent of $A$. For very small $A$ and $a_{r}$, the situation is just like the case of flow in a parallel-plate channel without any beams.

Figure 8 shows the dependence of $f R e$ on $a_{\mathrm{r}}$ for smaller $A$. It is noted that the predicted $f R e$ of the present study approaches the theoretical results obtained from a parallel-plate channel [5] for a smaller $A$ as $a_{r}$, tends to zero.

\section{CONCLUSION}

The following conclusions can be drawn from the foregoing results of this study:

(1) In addition to $f R e$ values, an increase in the beams' cross-sectional area will lead to a decrease in the mean velocities and volumetric flow rates of the fluid in the channel.

(2) For a fixed cross-sectional area of the beam, an increase in the height, $b$, will bring out an increase in the mean velocity and volumetric flow rate of the fluid, but a decrease in the fRe.

(3) The coefficients $A_{n}$ and $B_{m}$ in velocities $w_{1}(x, y)$ and $w_{2}(x$, $y$ )of the semi-analytical solution in this study can be more easily computed by the point match methods than that by the direct integral methods.

(4) The predicted $f R e$ of present study approaches the theoretical results obtained from a parallel-plate channel for smaller $A$ and $a_{r}$.

\section{NOMENCLATURE}

dimensionless half height of channel

$a_{r} \quad$ aspect ratio parameter $\left[=\frac{b /(1-c)}{1 / A}\right]$

A dimensionless area of cross section of a beam $[=b(1-c)]$

$A_{n} \quad$ coefficient of the function (Eq. (8))

$b$ dimensionless half height of the beam

$B_{m} \quad$ coefficient of the function (Eq. (9))

$c$ dimensionless half spacing between width of the beams

$D_{h} \quad$ hydraulic diameter, $=\frac{4[a-b(1-c)] H}{2+b-c}(\mathrm{~m})$ 
$f \cdot R e$ friction factor-Reynolds number product

$H \quad$ half span of the selected domain, (m)

$P \quad$ pressure, $\left(\mathrm{N} / \mathrm{m}^{2}\right)$

Re Reynolds number $\left[=W_{z} D_{h} / v\right]$

$W_{z} \quad$ velocity in the $Z$-direction, $(\mathrm{m} / \mathrm{s})$

$w \quad$ dimensionless velocity in the $\mathrm{z}$-direction $\left[=-W_{z} \mu d z /\right.$ $\left.H^{2} d p\right]$

$w_{\text {mean }}$ dimensionless mean velocity of the flow

$x$

$X$

horizontal axis $(\mathrm{m})$

dimensionless vertical axis $[=Y / H]$

vertical axis (m)

dimensionless axis normal to the paper

normal axis $(\mathrm{m})$

viscosity $\left(\mathrm{N}-\mathrm{s} / \mathrm{m}^{2}\right)$

\section{APPENDIX}

From (19) and the definition $A=b(1-c)$, it can be easily derived as

$$
a_{r}=b^{2}
$$

So, the dimensionless cross-sectional area of beams can be rewritten as

$$
A=\sqrt{a_{r}}(1-c)
$$

After some manipulation, Eq. (A2) turns to

$$
c=1-\frac{A}{\sqrt{a_{r}}}
$$

Since the dimensionless half spacing between width of the beams, $c$, must always be positive, the range of $A$ is obtained

as

$$
\sqrt{a_{r}} \geq A>0
$$

\section{REFERENCES}

1. Bahrami, M., Yovanovich, M. M., and Culham, J. R., "Pressure drop of fully-developed, laminar flow in microchannels of arbitrary crosssection," Journal of Fluids Engineering, Vol. 128, pp. 1036-1044 (2006).

2. Chen, J.-H., Pritchard, W. G., and Tavener, S. J., "Bifurcation for Flow Past a Cylinder between Parallel Planes," Journal of Fluid Mechanics, Vol. 284, pp. 23-41 (1995).

3. Cheng, K. C., "Analog solution of laminar heat transfer in noncircular ducts by moiré method and point-matching," Journal of Heat Transfer, Vol. 88, pp. 175-182 (1966).

4. Denton, J. A. and Beveridge, J. R., "An algorithm for projective point matching in the presence of spurious points," Pattern Recognition, Vol. 40, No. 2, pp. 586-595 (2007).

5. Holman, J. P., Heat Transfer, 8th edition, McGraw Hill Company, Singapore, p. 293 (2001).

6. Hsieh, T.-C. and Chen, J.-H., "Emergence of attached recirculating eddy for flow around a circular cylinder asymmetrically placed in a channel," Journal of Marine Science and Technology, Vol. 14, No. 3, pp. 147-154 (2006).

7. Skea, D., Barrodale, I., Kuwahara, R., and Poeckert, R., "A control point matching algorithm," Pattern Recognition, Vol. 26, No. 2, pp. 269-276 (1993).

8. Sparrow, E. M. and Loeffler, A. L., "Longitudinal laminar flow between cylinders arranged in regular array," AIChE Journal, Vol. 5, pp. 325-330 (1959).

9. Trogdon, S. A. and Joseph, D. D., "Matched eigenfunction expansions for a slow flow over a slot," Journal of Non-Newtonian Fluid Mechanics, Vol 10, pp. 185-213 (1982).

10. Wang, C. Y., "Parallel flow between corrugated plates," Journal of Engineering Mechanical Division, Vol. 102, pp. 1088-1090 (1976).

11. Wang, C. Y., "Flow in a channel with longitudinal ribs," Journal of Fluids Engineering, Vol. 116, pp. 233-237 (1994).

12. Zarling, J. P., "An analysis of laminar flow and pressure drop in complex shaped ducts," Journal of Fluids Engineering, Vol. 21, pp. $702-706$ (1976). 